\title{
Circulatory collapse after sheath removal in transfemoral transcatheter aortic valve implantation
}

\author{
Shihoko Iwata ${ }^{1}$, Choko Kume ${ }^{1}$, and Makoto Ozaki ${ }^{1}$ \\ ${ }^{1}$ Tokyo Women's Medical University
}

November 24, 2020

\begin{abstract}
Retroperitoneal hemorrhage is a rare but severe vascular access-related complication of transfemoral transcatheter aortic valve implantation. The diagnosis is often delayed because of nonspecific clinical presentations. Although the best management protocol is still controversial, endovascular treatment or embolization should be chosen in uncontrollable ongoing bleeding.
\end{abstract}

Circulatory collapse after sheath removal in transfemoral transcatheter aortic valve implantation

Shihoko Iwata ${ }^{\mathrm{a}}$, Choko Kume ${ }^{\mathrm{a}}$, and Makoto Ozaki ${ }^{\mathrm{a}}$

${ }^{a}$ Department of Anesthesiology, Tokyo Women's Medical University Hospital, Tokyo, Japan.

Correspondence to Shihoko Iwata, MD, PhD. Department of Anesthesiology, Tokyo Women's Medical University Hospital, 8-1 Kawada-cho, Shinjuku-ku, Tokyo, Japan, 162-8666

Tel: +81-3-3353-8111; e-mail:shk_wt_0204@ybb.ne.jp

Keywords

transcatheter aortic valve implantation, retroperitoneal hemorrhage, vascular access-related complication, hemodynamic instability, perforation

Key Clinical Message

Retroperitoneal hemorrhage is a rare but severe vascular access-related complication of transfemoral transcatheter aortic valve implantation. In cases of uncontrollable ongoing bleeding, endovascular treatment or embolization should be chosen.

Case Presentation

An 87-year-old small woman underwent transfemoral transcatheter aortic valve implantation (TF-TAVI) uneventfully and was transferred to an intensive care. However, hemodynamic instability became gradually obvious, and the abdomen progressively became swollen. Contrast-enhanced computed tomography revealed free fluid in the retroperitoneal and intraabdominal cavities, suggestive of retroperitoneal hemorrhage (RPH), that perforated the abdominal cavity with extravasation of contrast material (arrow) (Fig. 1a). The injured site of the external iliac artery (arrow) was confirmed using digital subtraction angiography (Fig. 1b) and repaired with a cover stent.

$\mathrm{RPH}$ is a rare but severe complication of TF-TAVI and has been reported in $0 \%-2.2 \%$ of cases. ${ }^{1}$ It is associated with iliofemoral arterial injuries, comprising the most frequent vascular access-related complication, ${ }^{2}$ of which small vessel dimensions, calcification, and center experience are the major predictors. ${ }^{1}$ The diagnosis 
of RPH is often delayed because of nonspecific clinical presentations such as flank, abdominal, back pain, and/or progressive hemodynamic instability. ${ }^{1,2}$ Although the best management protocol for RPH is still controversial, conservative management should only be applied in stable patients who require hemostasis. In cases of uncontrollable ongoing bleeding, endovascular treatment or embolization should be chosen. Open surgical intervention is rarely required. ${ }^{2}$ If treated inappropriately, the mortality remains high. ${ }^{2}$

Conflict of Interest

None declared.

Author Contributions

SI: wrote the initial draft of the manuscript and procured the clinical images. All the authors, SI, CK, and MO: made a substantial contribution to the preparation of this manuscript and approved the final version for submission.

\section{References}

Toggweiler S, Leipsic J, Binder RK, et al . Management of vascular access in transcatheter aortic valve replacement: part 2: Vascular complications. JACC: Cardiovascular Interventions 2013; 6 : 767-776.

Chan YC, Morales JP, Reidy JF, et al . Management of spontaneous and iatrogenic retroperitoneal haemorrhage: conservative management, endovascular intervention or open surgery? TheInternational Journal of Clinical Practice 2008; 62 : 1604-1613.
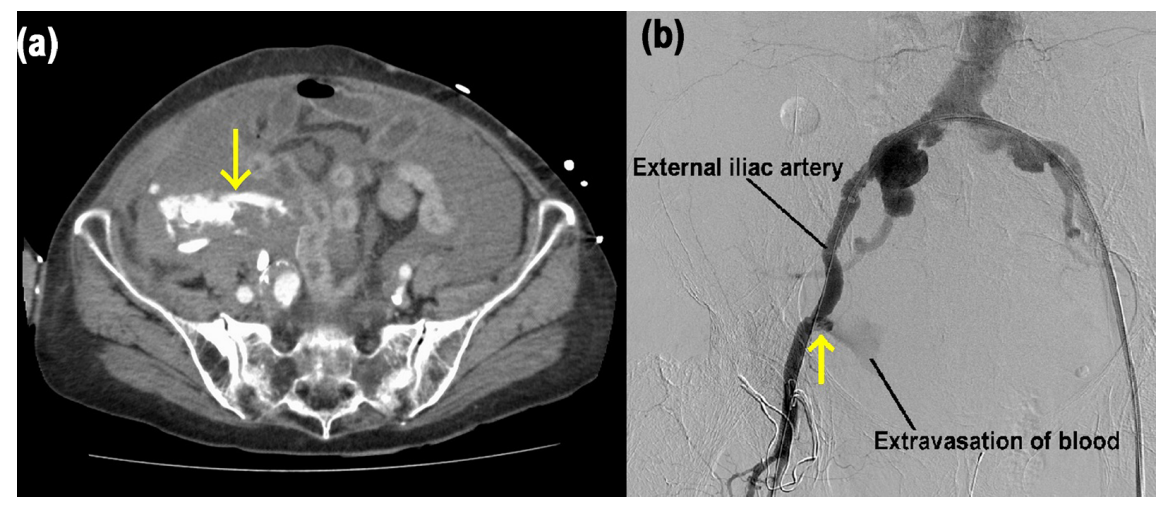